\title{
ACQuired Hemophilia A: A Case Report
}

\author{
Gunjan Shah, MD, Ajay Kandra, MD, Douglass A. Drelich, $M D$
}

\section{Case Report}

A 61-year-old African American male, with a past medical history of asthma and benign prostatic hypertrophy, presented from an outside hospital with complaints of hematuria and hematemesis. He had initially noted increased bruising two months prior to admission. He also had symptoms suggestive of a non-traumatic thigh hematoma the month prior. He developed hematuria one month prior to admission and underwent an outpatient cystoscopy one week prior to admission which was non-diagnostic. Cystoscopy was repeated on the day of admission localizing the hemorrhage to his right kidney with a biopsy specimen suggesting a low-grade papillary neoplasm. The patient subsequently developed hemetemesis. Emergent esophagogastroduodenoscopy (EGD) at the outside hospital revealed a Mallory-Weiss tear. Epinephrine was injected with initial hemostasis. EGD was repeated after transfer due to recurrent hematamesis and clips were placed to control the hemorrhage. The patient experienced continued hematuria requiring repeat cystoscopy with laser ablation, as well as biopsy of the right ureter and right mid-pole infundibulum. However, multiple repeated biopsies failed to confirm the presence of malignancy.

On admission, he was afebrile, with a heart rate of $94 \mathrm{bpm}$, blood pressure of $142 / 88 \mathrm{~mm} \mathrm{Hg}$, and oxygen saturation of $100 \%$ on room air. His physical exam was within normal limits .His initial hemoglobin was $7.6 \mathrm{~g} / \mathrm{dL}$, white blood cell count of 9,700 cells per cubic millimeter $(\mathrm{cmm})$, and platelet count 138,000 cells per cubic millimeter. Coagulation studies revealed an elevated activated partial thromboplastin time (aPTT) of 76.5 seconds. An aPTT mixing study was performed with the aPPT correcting to 34.9 seconds (normal 23.6-35 seconds). Incubation was not performed. Additional laboratory evaluation demonstrated factor VIII $<0.01 \mathrm{U} / \mathrm{ml}$ (normal $0.52-1.43 \mathrm{U} / \mathrm{ml}$ ) and a factor VIII antibody was detected with a measured titer of 33.3 Bethesda Units (BU). Von Willebrand antigen and activity levels were in the normal ranges.

Given the undetectable Factor VIII level, lack of prior history of a bleeding diathesis, and the presence of an inhibitor with a high titer level, a diagnosis of Acquired Hemophilia A (AHA) was made.

The initial management strategy was to control the acute bleeding and eradicate the inhibitor. Hemostasis was initially obtained with recombinant Factor VIIa (rFVIIa) at $90 \mathrm{mcg} /$ kg every 3-4 hours episodically for major bleeding for 6-12 hours and prior to planned invasive procedures. However due to a decrease in clinical response during the admission, it was switched to activated Prothrombin Complex Concentrate (aPCC), specifically factor eight inhibitor bypass activity (FEIBA).
Immunosuppression was initiated with $60 \mathrm{mg}$ of prednisone daily. ${ }^{3,30,31}$ He continued to experience severe hematuria requiring continuous bladder irrigation and multiple cystoscopies for clot evacuation and laser fulguration. Five days after initiation of prednisone, the patient failed to show significant clinical improvement, which warranted replacement with another type of immunosuppressive therapy. Pulsed high-dose dexamethasone $40 \mathrm{mg}$ (Dex) ${ }^{45-49}$ and Rituximab ${ }^{35-40}$ were administered for a period of four days. However, the patient had a grade 4 reaction to the Rituximab consistent with anaphylaxis requiring cessation of the treatment. He then received intravenous immunoglobulin (IVIG) for two days ${ }^{25-27}$ without any clinical response.

Given his inadequate response to standard and high dose steroids and IVIG, and a factor VIII level of $2 \%$ with an inhibitor titer of $40 \mathrm{BU}$, oral cyclosporine was initiated on hospital day 22 and titrated to maintain a therapeutic goal of $200-400 \mathrm{ng} / \mathrm{ml} .^{41-44}$ His hematuria gradually improved, and he required no further transfusions.

Over the course of his 36-day hospitalization he received a total of 22 units of pRBC, 9 units of FFP, 34 doses of rFVIIa, and 6 doses of FEIBA. He was discharged on a therapeutic dose of oral cyclosporine with a Factor VIII level of $4 \%$ and an inhibitor titer of $57.1 \mathrm{BU}$ at the time of discharge (Graph 1).

He was evaluated weekly following discharge during which he did not have any major hemorrhage. His cyclosporine dose was titrated based on trough levels. He continued pulsed, high-dose dexamethasone for 4 consecutive days every 28 days. His Factor VIII level demonstrated gradual improvement during this period and inhibitor levels gradually decreased. Following the fourth cycle of high-dose dexamethasone, his Factor VIII level rose to $29 \%$ and his inhibitor level became undetectable. Dexamethasone was discontinued following the fourth cycle after obtaining an undetectable inhibitor level. Cyclosporine weaning was initiated following two consecutive factor levels greater than $80 \%$.

\section{Discussion}

Acquired Hemophilia A (AHA) is a rare condition in which autoantibodies, usually of the IgG class, are produced against Factor VIII. This results in low plasma Factor VIII levels. Its incidence ranges from $0.2-4$ cases/million/year according to various reports. ${ }^{1,2,3,4,5}$ However, given the complexity of diagnosis, the condition may be under diagnosed. ${ }^{11,12,15}$ The incidence increases with age with a peak incidence in the $7^{\text {th }}$ and $8^{\text {th }}$ decade of life. There is a small peak in the $3^{\text {rd }}$ decade likely corresponding to postpartum inhibitors. ${ }^{3}$ The mortality rate is high, ranging from $8 \%-22 \%$. This is related to severe hemorrhage that can occur in $85 \%-90 \%$ of the patients. ${ }^{3,4}$ Approximately half of the cases are associated with underlying conditions including pregnancy, autoimmune disorders, malignancy, medications, dermatologic conditions, inflammatory bowel disease, and 


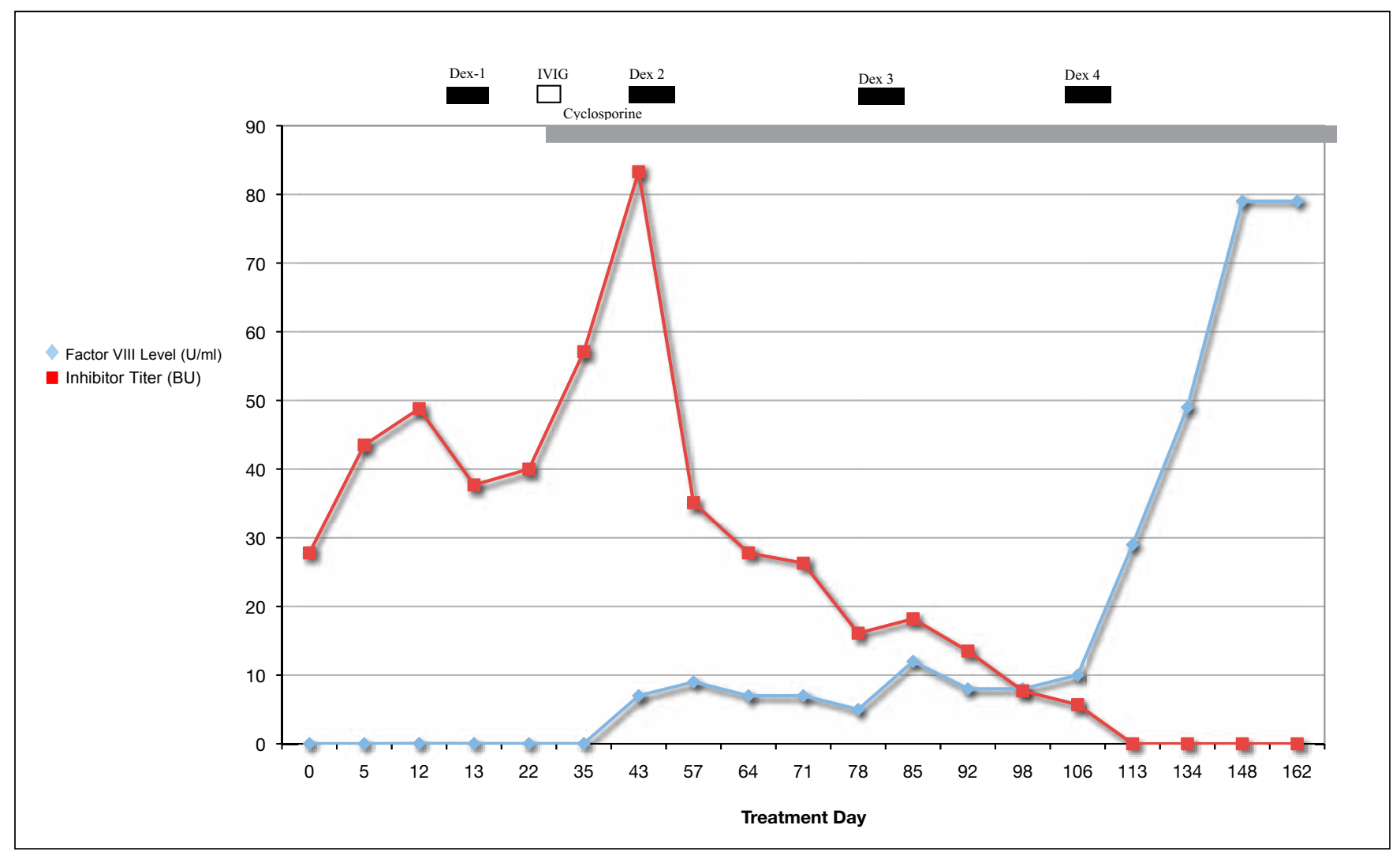

Figure 1. Factor VIII Level and Inhibitor Titer by Treatment Day

infections such as hepatitis B and C. The other half occur without evidence of a coexisting disorder (idiopathic)., ${ }^{3,6,7,8,9,10}$

This condition commonly presents with mucosal bleeding (including epistaxis, gastrointestinal, and genitourinary), ecchymosis, and soft tissue hemorrhage including retroperitoneal bleeding. In contrast to congenital Hemophilia A, hemarthrosis is rare. ${ }^{3,4}$

The diagnosis is confirmed by detection of a prolonged aPTT which does not correct by 1:1 mixing with normal plasma (aPTT mixing study). Occasionally, there is immediate correction but after incubation for 2 hours at $37^{\circ} \mathrm{C}$ there is reversal of the correction. Factor VIII levels are markedly reduced, and anti-FVIII antibodies are detectable.

The primary goals of treatment for AHA are to control acute bleeding and to suppress the autoantibody. Antibody eradication often requires treatment of the instigating condition when present. AHA can represent a transfusion emergency requiring intensive transfusion support. ${ }^{13}$ In patients with high titer inhibitors ( $>5$ $\mathrm{BU})$, bypassing agents, such as FEIBA and rVIIa, are the mainstay of therapy for acute hemorrhage. ${ }^{4-21}$ The recommended dose of FEIBA, ranges between 50-100 U/kg administered every 6-12 hours, not to exceed a single dose of $100 \mathrm{U} / \mathrm{kg}$ or a daily dose of
$200 \mathrm{U} / \mathrm{kg} .{ }^{3,14,15,16}$ In one study, FEIBA controlled hemorrhage in $86 \%$ of patients. ${ }^{14}$ The recommended dose of rVIIa ranges from 90 to $120 \mathrm{mcg} / \mathrm{kg}$ every 2-3 hours. ${ }^{17-21}$ A pooled analysis of data of 139 patients from different sources reported an efficacy rate of $88 \% .{ }^{17}$ Venous thromboembolic disease (VTE) is an adverse event associated with both of these agents.

Factor VIII in large doses should be considered in patients known to have low-titer inhibitors $(<5 \mathrm{BU})$. Porcine Factor VIII from pooled plasma, while not currently available in the US, has been used historically to increase plasma factor VIII levels, and it takes advantage of the minimal antigenic cross reactivity with human Factor VIII inhibitors. ${ }^{22,23,24} \mathrm{~A}$ recombinant porcine factor VIII, B-domain deleted (OBI-1) has recently been tested in a clinical trial in the US (50).

A number of immunosuppressive therapies have been used to control inhibitors. However, current treatment is based on small, uncontrolled, single-center cohorts and meta-analysis. Corticosteroids and cytotoxic drugs, used either alone or in combination, have been regarded as the mainstay of therapy. An alternate modality of inhibitor suppression is IVIG; however, this has generally been less effective. ${ }^{11,25,26,27}$ Removal of the inhibitor via an immunoadsorption process has been 
attempted. ${ }^{3,28,29}$ But this process requires central venous access, specialized equipment and training, and close FVIII monitoring.

Oral steroids used alone at $1 \mathrm{mg} / \mathrm{kg}$ prednisone equivalents per day for 3-6 weeks can cause remission in one third to one half of patients with acquired hemophilia. ${ }^{3,16,30,31}$ Cyclophosphamide has been used as initial therapy and in refractory patients with high-titer inhibitors ( $>5 \mathrm{BU}$ ) leading to higher remission rates

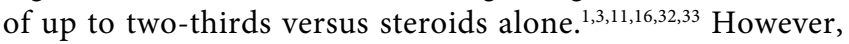
a 2-year national surveillance report of acquired hemophilia A by the UK Haemophilia Centre Doctors' Organization concluded that there was no difference in inhibitor eradication or mortality between patients treated with steroids alone or with a combination of steroids and cytotoxic agents. ${ }^{11}$

Several reports have demonstrated the effectiveness of Rituximab in acquired hemophilia A. The general high level of tolerance of this drug has led to initial use of this treatment. However, there is currently no data to establish its superiority over other treatments. The dose typically given is $375 \mathrm{mg} / \mathrm{m} 2$ infused weekly for 4 weeks and administered concomitantly with other immunosuppressive drugs. ${ }^{34-39}$

Cyclosporine (CsA) has also been used for inhibitor suppression. ${ }^{40-43}$ Use of CsA requires monitoring drug levels to ensure efficacy and prevent toxicity. However, its use is limited due to availability of other strategies such as cytotoxic drugs and Rituximab.

We describe a case of AHA with high titer antibody that experienced clinical deterioration on initial monotherapy with prednisone. The patient was unable to tolerate Rituximab due to anaphylaxis and was also refractory to IVIG.

We report a novel strategy of combining Cyclosporine with pulsed, high dose dexamethasone at a dose of 40mg daily for four consecutive days every 28 days. Pulsed dose dexamethasone is an acceptable modality of immunosupression and has been used for the treatment of Idiopathic Thrombocytopenic Purpura (ITP), another immune-mediated hematological disorder. ${ }^{44-48}$ In general it is tolerated well, without producing the long term effects of steroid toxicity. No randomized trials exist comparing the efficacy and tolerability of pulse dose dexamethasone with longer duration steroids. However, we have experienced good results in our institution for the treatment of ITP, and this has become our preferred first line therapeutic modality. The patient tolerated this regimen extremely well and went into complete remission with undetectable antibody titers after the fourth cycle of pulse dexamethasone. He is currently in remission and is being weaned from cyclosporine.

\section{References}

1. Delgado J,Yimenez-YusteV, Hernandez-Navarro F,Villar A. Acquired haemophilia: review and meta-analysis focused on therapy and prognostic factors. Br J Haematol 2003;121:21-35.

2. Collins P, Macartney N, Davies B, Lees S, Giddings J, Maier R. A population based, unselected, consecutive cohort of patients with acquired haemophilia A.
Br J Haematol 2004;124:86-90

3. Massimo Franchini and Giuseppe Lippi. How I treat Acquired factor VIII inhibitors. Blood 2008; 112: 250-255.

4. Green D, Lechner K. A survey of 215 nonhemophilic patients with inhibitors to factor VIII. Thromb Haemost 1981; 45:200-203.

5. Sallah S, Nguien NP, Abdallah JM, Hanrahan LR. Acquired hemophiliain patients with hematologic malignancies. Arch Pathol Lab Med 2000;124:730-4.

6. Massimo Franchini, Giovanni Targher, Franco Manzato. Acquired factor VIII inhibitors in oncohematology: A systematic review. Crit Rev Oncol Hematol 2008; 66: 194-199.

7. Sallah S,Wan JY. Inhibitors against factor VIII in patients with cancer. Cancer 2001;91:1067-74.

8. Meiklejohn DJ,Watson HG. Acquired haemophilia in association with organspecific autoimmune disease. Haemophilia 2001;7:523-5.

9. Hultin MB. Acquired inhibitors in malignant and nonmalignant disease states. Am J Med 1991;91:9-13

10. Sallah S, Nguien NP, Abdallah JM, Hanrahan LR. Acquired hemophilia in patients with hematologic malignancies. Arch Pathol Lab Med 2000;124:730-4.

11. Peter W. Collins, Sybil Hirsch, Trevor P. Baglin. Acquired hemophilia A in the United Kingdom: a 2-year national surveillance study by the United Kingdom Haemophilia Centre Doctors' Organisation. Blood 2007;109:1870-1877.

12. Paul Giangrande. Acquired Hemophilia. Published by the World Federation of Hemophilia (WFH) 2005.

13. Giuseppe Tagariello \& Roberto Sartori \& Paolo Radossi. Intensive blood transfusion support in acquired hemophilia A. Ann Hematol 2007; 86:229-230.

14. Sallah S. Treatment of acquired haemophilia with factor eight inhibitor bypassing activity. Haemophilia 2004; 10:169-173.

15. P.A. Holme, F. Brosstad, G.E.Tjonnfjord. Acquired haemophilia: management of bleeds and immune therapy to eradicate autoantibodies. Haemophilia $2005 ; 11$ : 510-515.

16. Brian Barnett, Rebecca Kruse-Jarres and Cindy A. Leissinger. Current management of acquired factor VIII inhibitors. Curr Opin Hematol 2008;15:451-455.

17. Sumner MJ, Geldziler BD, Pedersen M, Seremetis S. Treatment of acquired haemophilia with recombinant activated FVII: a critical appraisal. Haemophilia 2007; 13:451-461.

18. Hay CRM, Negrier C, Ludlam CA. The treatment of bleeding in acquired haemophilia with recombinant factor VIIa: a multicenter study. Thromb Haemost 1997; 78:1463-1467.

19. Parameswaran R, Shapiro AD, Gill JC, Kessler CM. Dose effect and efficacy of rFVIIa in the treatment of haemophilia patients with inhibitors: analysis from the Hemophilia and Thrombosis Research Society Registry. Haemophilia 2005; 11: $100-6$.

20. Abshire T, Kenet G. Recombinant factor VIIa: review of efficacy, dosing regimens and safety in patients with congenital and acquired factor VIII or IX inhibitors. J Thromb Haemost 2004;2:899-909.

21. Baudo F, de Cataldo F, Gaidano G. Treatment of acquired factor VIII inhibitor with recombinant activated factor VIIa: data from the Italian registry of acquired hemophilia. Haematologica. 2004;89:759-761.

22. Huang YW, Saidi P, Philipp C. Acquired factor VIII inhibitors in nonhaemophilic patients: clinical experience of 15 cases. Haemophilia 2004; 10:713-721.

23. Morrison AE, Ludlam CA, Kessler C. Use of porcine factor VIII in the treatment of patients with acquired hemophilia. Blood 1993; 81:1513-1520.

24. Morel Rubinger, Donald S. Houston, Nora Schwetz. Continuous infusion of porcine factor VIII in the management of patients with factor VIII inhibitors. Am J Hematol 1998;56:112 - 118.

25. Dykes AC, Walker ID, Lowe GD, Tait RC. Combined prednisolone and intravenous immunoglobulin treatment for acquired factor VIII inhibitors: a 2-year review. Haemophilia. 2001;7:160- 163.

26. Schwartz RS, Gabriel DA, Aledort LM, Green D, et al. A prospective study of treatment of acquired (autoimmune) factor VIII inhibitors with high-dose intravenous gammaglobulin. Blood 1995; 86:797-804.

27. Crenier L, Ducobu J, des Grottes JM, Cerny J, Deunoit C, Capel P. Low response 
to high-dose intravenous immunoglobulin in the treatment of acquired factor VIII inhibitor. Br J Haematol 1996;95:750-753.

28. Rivard GE, St Louis J, Lacroix S, Champagne M, et al. Immunoadsorption for coagulation factor inhibitors: a retrospective critical appraisal of 10 consecutive cases from a single institution. Haemophilia 2003; 9:711-716.

29. Freedman J, Rand ML, Russell O, Davis C, et al. Immunoadsorption may provide a cost-effective approach to management of patients with inhibitors to FVIII. Transfusion 2003; 43:1508-1513.

30. Spero JA, Lewis JH, Hasiba U. Corticosteroid therapy for acquired F VIII:C inhibitors. Br J Haematol 1981; 48: 635-42.

31. Green D, Rademaker AW, Briet E. A prospective, randomized trial of prednisone and cyclophosphamide in the treatment of patients with factor VIII autoantibodies. Thromb Haemost 1993; 70:753-757.

32. Collins PW. Treatment of acquired hemophilia A. J Thromb Haemost 2007;5:893-900

33. Boggio LN, Green D. Acquired hemophilia. Rev Clin Exp Hematol 2001; 5:389-404;

34. Stasi R, Brunetti M, Stipa E, Amadori S. Selective B-cell depletion with rituximab for the treatment of patients with acquired hemophilia. Blood 2004; 103:44244428 .

35. Abdallah A, Coghlan DW, Duncan EM, Chunilal SD, et al. Rituximab-induced long-term remission in patients with refractory acquired hemophilia. J Thromb Haemost 2005; 3:2589-2590.

36. Wiestner A, Cho HJ, Asch AS, et al. Rituximab in the treatment of acquired factor VIII inhibitors. Blood 2002; 100:3426-3428.

37. Garvey B. Rituximab in the treatment of autoimmune haematological disorders. Br J Haematol 2008; 141:149-169.

38. Franchini M. Rituximab in the treatment of adultacquired hemophilia A: a systematic review. Crit Rev Oncol Hematol 2007;63:47-52.
39. Aggarwal A, Grewal R, Green RJ, et al. Rituximab for autoimmune haemophilia: a proposed treatment algorithm. Haemophilia 2005; 11:13-9.

40. Schulman S, Langevitz P, Livneh U, Mortinowitz U, Seligsohn U, Varon D. Cyclosporine therapy for acquired factor VIII inhibitor in a patient with systemic lupus erythematosus. Thromb Haemost 1996; 76: 344-6.

41. Pfliegler G, Boda Z, Harsfalvi J, et al. Cyclosporin treatment of a woman with acquired haemophilia due to factor VIII:C inhibitor. Postgrad Med J 1989; 65:400.

42. Petrovic M, Derom E, Baele G. Cyclosporine treatment of acquired hemophilia due to factor VIII antibodies. Haematologica. 2000;85:895-896.

43. Au WY, Lam CC, Kwong YL. Successful treatment of acquired factor VIII inhibitor with cyclosporin. Haemophilia. 2004;10:98-100.

44. Caulier MT, Rose C, Roussel MT, Fenaux P. Pulsed high-dose dexamethasone in refractory chronic idiopathic thrombocytopenic purpura: a report on 10 cases. $\mathrm{Br}$ J Haematol 1995 Oct;91(2):477-9.

45. Cheng Y, Wong RS, Soo YO, Chui CH, Lau FY, Chan NP, Wong WS, Cheng G. Initial treatment of immune thrombocytopenic purpura with high-dose dexamethasone. N Engl J Med. 2003 Aug 28;349(9):831-6.

46. Bulvik S, Ben-Tal O, Shimoni Z, Eldor A. Pulsed high-dose dexamethasone in resistant immune thrombocytopenic purpura. Harefuah. July 1996;131(12):18-20, 71 .

47. Wali YA, Al Lamki Z, Shah W, Zacharia M, Hassan A. Pulsed high-dose dexamethasone therapy in children with chronic idiopathic thrombocytopenic purpura. Pediatr Hematol Oncol 2002 Jul - Aug;19(5):329-35.

48. Chen JS, Wu JM, Chen YJ, Yeh TF. Pulsed high-dose dexamethasone therapy in children with chronic idiopathic thrombocytopenic purpura. J Pediatr Hematol Oncol 1997 Nov-Dec;19(6):526-9. 